\title{
THE RATE OF ANGULAR MOMENTUM LOSS FROM CLOUD CORES
}

\author{
Takenori Nakano \\ Department of Physics, Kyoto University \\ Sakyo-ku, Kyoto 606, Japan
}

\section{Introduction}

The angular momentum is one of the major obstacles to the contraction of interstellar clouds. An efficient process of removing the angular momentum from the cloud is via transport along the magnetic field lines to the ambient medium. When the magnetic field is nearly uniform and the direction of the field lines is parallel to the rotation axis, the spin-down time of the cloud is given by $\sigma / 2 \rho V_{\mathrm{A}}$, where $\sigma$ is the column density of the cloud along the field lines, and $\rho$ and $V_{\mathrm{A}}$ are the density and the Alfvén velocity, respectively, in the ambient medium (Ebert et al. 1960; Mouschovias \& Paleologou 1980). However, this is for a cloud with weak gravity. Because a cloud with strong gravity has contracted dragging the field lines, the ambient field is considerably distorted from uniformity. The spin-down time of such a cloud is shorter than given above (Gillis, Mestel \& Paris 1974, 1979).

A cloud can be in magnetohydrostatic equilibrium when its mass is smaller than the critical mass $M_{\mathrm{cr}} \approx \Phi / \pi G^{1 / 2}$, where $G$ is the gravitational constant and $\Phi$ is the magnetic flux through the cloud (Mestel 1965, 1966; Strittmatter 1966; Nakano 1981, 1984). As shown by Nakano $(1979,1982,1983)$, the process referred to alternatively as 'ambipolar diffusion' or 'plasma drift' (Mestel \& Spitzer 1956) induces highly non-homologous quasistatic contraction of such a cloud: a high-density core soon appears and finally contracts leaving the outer part nearly unchanged. Magnetic braking of such a core is important because this must be one of the processes leading to star formation. Although the magnetic field may be nearly uniform very far from the cloud, there is an intermediate zone where the field lines anchored to the core are nearly radial and the angles between the field lines and the symmetry axis are very small (Nakano 1979, 1982, 1983) as shown schematically in Fig. 1. We investigate the transport of the angular momentum of the core to this intermediate zone assuming that the sphere (dashed circle) in Fig. 1 is sufficiently large and the core is a rigid rotator.

\section{The Model and Formulation}

We consider an axisymmetric system composed of a cloud and an ambient medium. Because we can take the magnetic field as being well frozen into the ambient gas (Nakano \& Umebayashi 1980, 1986a, b; Nakano 1984, 1988), we have for the 
magnetic field $\mathbf{B}$ and the velocity $\mathbf{v}$ of the gas

$$
\frac{\partial \mathbf{B}}{\partial t}=\nabla \times(\mathbf{v} \times \mathbf{B})
$$

The torque equation for the gas is given by

$$
\mathbf{e}_{z} \frac{\partial}{\partial t}\left(\rho \varpi v_{\varphi}\right)=\varpi \mathbf{e}_{\varpi} \times \frac{1}{c}(\mathbf{j} \times \mathbf{B})
$$

where $\rho$ is the density of the gas, $v_{\varphi}$ is the azimuthal component of the velocity, $\varpi$ is the distance from the symmetry axis, $\mathbf{e}_{z}$ and $\mathbf{e}_{\varpi}$ are the unit vectors along the symmetry axis and in the $\varpi$-direction, respectively, $c$ is the light velocity, and $\mathbf{j}=(c / 4 \pi) \nabla \times \mathbf{B}$ is the electric current density.

We adopt spherical polar coordinates $(r, \theta, \varphi)$ and assume $B_{\theta}=0$ and the pure rotatory motion. This is a good approximation at least when the rotational velocity $v_{\varphi}$ is much smaller than the Alfvén velocity. At least at $\theta \ll 1$ we can regard that $B_{r}$ is independent of $\theta$ and then the magnetic field is torque-free before the torsional Alfvén wave arrives. Equations (1) and (2) give a wave equation for $\Omega=v_{\varphi} / r \sin \theta$,

$$
\frac{\partial^{2} \Omega}{\partial t^{2}}=\frac{B_{r}^{2}}{4 \pi \rho} \frac{\partial^{2} \Omega}{\partial r^{2}}
$$

We assume that the cloud core is supported mainly by the magnetic field or by the thermal pressure and neglect contraction due to angular momentum loss. The thickness of the core along the field lines is usually smaller than its size perpendicular to them, and so the field lines cannot be radial near the core (Nakano 1979, 1982, 1983). However, this region with a non-radial field (between the dashed lines in Fig. 1 ) is rather narrow, and the torsional Alfvén wave passes through this region in a time much shorter than the spin-down time of the core. Hence the rotation of the gas in this region lags only slightly behind that of the core. We therefore assume that the layer between $z=-Z$ and $z=Z$, where the field lines are not radial, rotates rigidly with the core, and solve the wave propagation outside this layer. The torque equation for the core gives for the angular velocity of the core $\Omega_{\mathrm{cc}}$,

$$
\frac{d^{2} \Omega_{\mathrm{cc}}}{d t^{2}}=\frac{1}{2 \pi \sigma}\left(B_{r}^{2} \frac{\partial \Omega}{\partial r}\right)_{r=Z}
$$

where $\sigma$ is the column density of the core along the $z$-axis. The continuity of the field lines requires $\Omega_{\mathrm{cc}}(t)=\Omega(r=Z, t)$.

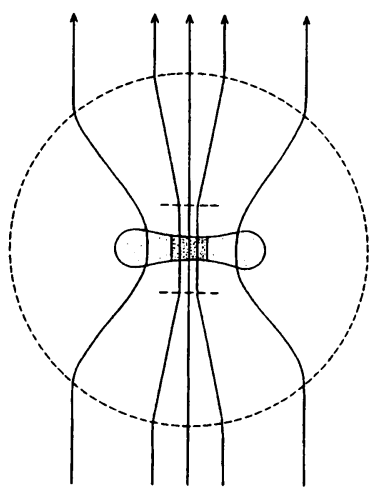

Fig. 1. A schematic diagram of the magnetic configuration around a cloud (shaded) and its core (heavily shaded). The magnetic field is uniform outside the sphere (dashed circle). The field lines anchored to the core are nearly radial and deviate only slightly from the symmetry axis in the intermediate zone outside the layer interposed between the dashed lines. We investigate the transport of the angular momentum of the core to the intermediate zone assuming that the sphere is sufficiently large. 
We assume that the ambient medium at $r \geq Z$ has a density distribution $\rho(r)=$ $\rho(Z)(r / Z)^{-n}$, where $n$ is a constant. We rewrite the equations in dimensionless forms. With $\zeta \equiv r / Z$ and $\tau \equiv t V_{\mathrm{A}}(Z) / Z$, where $V_{A}(Z)=B_{r}(Z) /[4 \pi \rho(Z)]^{1 / 2}$ is the Alfvén velocity at $r=Z$, equations (3) and (4) are rewritten as

$$
\frac{\partial^{2} \Omega}{\partial \tau^{2}}=\frac{1}{\zeta^{4-n}} \frac{\partial^{2} \Omega}{\partial \zeta^{2}} \quad \text { for } \zeta>1, \quad \frac{d^{2} \Omega_{\mathrm{cc}}}{d \tau^{2}}=\left.\frac{\rho(Z)}{\rho_{\mathrm{cc}}} \frac{\partial \Omega}{\partial \zeta}\right|_{\zeta=1},
$$

where $\rho_{\mathrm{cc}}=\sigma / 2 Z$. Equations (5) are solved with the initial conditions at $\tau=0$,

$$
\Omega=\frac{\partial \Omega}{\partial \tau}=0 \quad \text { for } \zeta>1, \quad \Omega_{\mathrm{cc}}=\Omega_{0}, \quad \frac{d \Omega_{\mathrm{cc}}}{d \tau}=0 .
$$

\section{Solutions}

By Laplace-transforming equations (5) with the above initial conditions we have

$$
s^{2} \widetilde{\Omega}=\frac{1}{\zeta^{4-n}} \frac{\partial^{2} \widetilde{\Omega}}{\partial \zeta^{2}} \quad \text { for } \zeta>1, \quad s^{2} \widetilde{\Omega}_{\mathrm{cc}}-s \Omega_{0}=\left.\frac{\rho(Z)}{\rho_{\mathrm{cc}}} \frac{\partial \widetilde{\Omega}}{\partial \zeta}\right|_{\zeta=1},
$$

where $\widetilde{\Omega}(\zeta, s)$ and $\widetilde{\Omega}_{\mathrm{cc}}(s)$ are the Laplace transforms of $\Omega(\zeta, \tau)$ and $\Omega_{\mathrm{cc}}(\tau)$, respectively. The first equation in (7) can be reduced to a modified Bessel equation. We solve equations ( 7$)$ with the boundary conditions $\widetilde{\Omega}(\zeta=\infty, s)=0$ and $\widetilde{\Omega}_{\mathrm{cc}}(s)=\widetilde{\Omega}(\zeta=1, s)$. The inverse transformation finally gives with $\nu=1 /(6-n)$

$$
\Omega(\zeta, \tau)=\int_{c-i \infty}^{c+i \infty} \frac{\tilde{\Omega}(\zeta, s)}{2 \pi i} e^{s \tau} d s=\int_{c-i \infty}^{c+i \infty} \frac{\Omega_{0} \zeta^{1 / 2} K_{\nu}\left(2 s \nu \zeta^{1 / 2 \nu}\right) e^{s \tau} / 2 \pi i}{s K_{\nu}(2 s \nu)+\left[\rho(Z) / \rho_{\mathrm{cc}}\right] K_{\nu-1}(2 s \nu)} d s .
$$

3.1. THE CASE OF $\nu=1 / 2 \quad(n=4)$

The integrand in equation (8) is a single-valued function on the complex $s$-plane and has a pole only at $s=-s_{1 / 2} \equiv-\rho(Z) / \rho_{\mathrm{cc}}$, and we have

$$
\Omega(\zeta, \tau)= \begin{cases}\Omega_{0} \exp \left[-s_{1 / 2}(\tau-\zeta+1)\right], & \text { for } \tau>\zeta-1, \\ 0, & \text { for } \tau<\zeta-1 .\end{cases}
$$

When the wave front arrives, the medium begins to rotate with $\Omega_{0}$, and afterwards the rotation decays with the timescale $s_{1 / 2}^{-1}$. The rotation of the cloud also decays with the same timescale because $\Omega_{\mathrm{cc}}(\tau)=\Omega(\zeta=1, \tau)=\Omega_{0} \exp \left(-s_{1 / 2} \tau\right)$.

\subsection{THE CASES OF $\nu$ BETWEEN 0 AND $1 / 2$}

We evaluate the integral approximately by assuming $\rho(Z) \ll \rho_{\mathrm{cc}}$. The integrand in equation (8) is a multivalued function. On the Riemann surface with $|\arg (s)| \leq \pi$ the integrand has only two poles at

$$
s_{ \pm}=s_{\nu} \exp [ \pm i \pi /(2-2 \nu)], \quad s_{\nu}=\left[\frac{\rho(Z)}{\rho_{\mathrm{cc}}} \nu^{2 \nu} \frac{\Gamma(1-\nu)}{\Gamma(1+\nu)}\right]^{1 /(2-2 \nu)} .
$$

We consider the rotation of the core by taking $\zeta=1$. The path of the integral on this Riemann surface can be decomposed into the circles around $s=s_{+}$and 
around $s=s_{-}$and the round path $\mathrm{C}$ along the negative real axis. We evaluate the integral along the path $\mathrm{C}$ numerically and find out an empirical formula which fits this result. We finally have

$$
\Omega_{\mathrm{cc}}(\tau) \approx \frac{\Omega_{0}}{1-\nu} \exp \left(s_{\nu} \tau \cos \frac{\pi}{2-2 \nu}\right) \cos \left(s_{\nu} \tau \sin \frac{\pi}{2-2 \nu}\right)-\frac{\Omega_{0} \nu /(1-\nu)}{\left(1+a_{\nu} s_{\nu} \tau\right)^{2-2 \nu}}
$$

for all $\tau \geq 0$. The constant $a_{\nu}$ is equal to $0.85,1.04$, and 1.48 for $\nu=1 / 6,1 / 4$, and $1 / 3$, respectively. The first term, which came from the circles, represents the oscillation with the amplitude decreasing exponentially in a timescale nearly equal to $s_{\nu}^{-1}$, and the second term, coming from the path $\mathrm{C}$, decays by the power law at $\tau \gtrsim s_{\nu}^{-1}$. Thus most of the initial angular momentum is lost in the time $s_{\nu}^{-1}$.

\subsection{THE PHYSICAL MEANING OF $s_{\nu}$}

Let $\zeta_{0}$ be the position where the moment of inertia of the medium in the magnetic tube anchored to the core inside $\zeta_{0}$ is equal to the moment of inertia of the core. Let $\tau_{\mathrm{A}}\left(\zeta_{0}\right)$ be the Alfvén-crossing time up to $\zeta_{0}$ from the core. Then we have

$$
s_{\nu} \tau_{\mathrm{A}}\left(\zeta_{0}\right) \approx 2[\Gamma(2-\nu) / \Gamma(\nu)]^{1 /(2-2 \nu)} .
$$

The right-hand side of this equation has values $0.69,0.80$, and 0.88 for $\nu=1 / 6,1 / 4$, and $1 / 3$, respectively. For $\nu=1 / 2$ we have $s_{1 / 2} \tau_{\mathrm{A}}\left(\zeta_{0}\right)=1.0$. Thus the spin-down time $s_{\nu}^{-1}$ is the time taken by the torsional Alfvén wave to cross a domain in the ambient medium with moment of inertia nearly equal to that of the cloud.

\section{Discussion}

The magnetic braking of contracted clouds has been investigated by several authors by assuming arbitrary ambient magnetic configurations. With an arbitrary configuration the magnetic field is not force-free and the configuration changes drastically before the wave front arrives from the cloud. Thus the treatment is usually not self-consistent. On the other hand, in our models with $\theta \ll 1$ we can adopt a forcefree initial field, and even after the wave front arrives, the $r$-and $\theta$-components of the magnetic force are negligible compared with the $\varphi$-component at least when the rotational velocity of the medium is much smaller than the Alfvén velocity. Thus our models are self-consistently built.

The spin-down time of a cloud embedded in a uniform magnetic field mentioned in Section 1 can be interpreted in the same way as $s_{\nu}^{-1}$. Thus for various magnetic configurations and density distributions in the ambient medium, and so probably universally, the cloud loses most of its initial angular momentum in the time taken by the torsional Alfvén wave to cross a region in the ambient medium with column density equal to that of the cloud.

\section{References}

Ebert, R., Hoerner, S. von \& Temesvary, S., 1960. Die Entstehung von Sternen durch Kondensation diffuser Materie, p. 315, Springer-Verlag, Berlin.

Gillis, J., Mestel, L. \& Paris, R. B., 1974. Astrophys. Space Sci., 27, 167.

Gillis, J., Mestel, L. \& Paris, R. B., 1979. Mon. Not. R. astr. Soc., 187, 311. 
Mestel, L., 1965. Quart. J. R. astr. Soc., 6, 265.

Mestel, L., 1966. Mon. Not. R. astr. Soc., 133, 265.

Mestel, L. \& Spitzer, L., Jr., 1956. Mon. Not. R. astr. Soc., 116, 503.

Mouschovias, T. Ch. \& Paleologou, E. V., 1980. Astrophys. J., 237, 877.

Nakano, T., 1979. Publ. astr. Soc. Japan, 31, 697.

Nakano, T., 1981. Prog. theor. Phys. Suppl., No. 70, p. 54.

Nakano, T., 1982. Publ. astr. Soc. Japan, 34, 337.

Nakano, T., 1983. Publ. astr. Soc. Japan, 35, 209.

Nakano, T., 1984. Fund. Cosmic Phys., 9, 139.

Nakano, T., 1988. Galactic and Extragalactic Star Formation, p. 111, eds Pudritz, R. E. \& Fich, M., Kluwer Academic Publishers.

Nakano, T. \& Umebayashi, T., 1980. Publ. astr. Soc. Japan, 32, 613.

Nakano, T. \& Umebayashi, T., 1986a. Mon. Not. R. astr. Soc., 218, 663.

Nakano, T. \& Umebayashi, T., 1986b. Mon. Not. R. astr. Soc., 221, 319.

Strittmatter, P. A., 1966. Mon. Not. R. astr. Soc., 132, 359.

KUNDT: When you consider cloud-core braking by transverse magnetic fields, why do you ignore the simultaneous braking within the stellar accretion disk (mediated, probably, by toroidal magnetic fields)?

NAKANO: I consider a cloud and its core contracted along the field lines. So the ambient disk is not connected magnetically to the core.

CAMENZIND: Alfvén torsional waves do not only mean angular momentum flow, but also an energy loss from the central core. Is there evidence for dissipation of this magnetic energy in the ambient medium?

NAKANO: The rotation energy of the core is converted into the rotational energy and the magnetic energy $\left(B_{\phi}^{2} / 8 \pi\right)$ of the ambient medium. As long as the ambient medium is inviscid and a perfect conductor, as assumed in my model, the energy is conserved. But if the viscosity and/or the electric resistivity are taken into account, the energy gradually dissipates.

MOUSCHOVIAS: As you have mentioned we (Mouschovias and Paleologou, 1980, Astrophys. J. 237, 877) first pointed out that the characteristic time $\left(\tau_{\|}\right)$for magnetic braking of an aligned rotator as a whole (i.e. ignoring short-lived transient effects within the rotator) is reliably represented by the time $\left(\tau_{I}\right)$ it takes for the torsional waves to affect a moment of inertia in the external medium equal to the moment of inertia of the rotator. In fact, we gave a relatively general expression for $\tau_{\text {I }}$ (see Mouschovias and Morton, 1985, Astrophys. J. 298, 190; also the earlier equivalent results by Mouschovias, 1983, in Solar and Stellar Magnetic Fields, ed. J.O. Stenflo, Reidel, Dordrecht, p. 479; or the review $1987 \mathrm{~b}$ in Physical Processes in Interstellar Clouds, eds. G.E. Morfill and M. Scholer, Reidel, Dordrecht, p. 491, Section 2.4) which is $\tau_{\|}=$ $I_{c l} / i_{\text {ext }} V_{A, e \times t}$, where $I_{c l}$ is half the moment of inertia of the rotator, $i_{\text {ext }}$ is the external-medium moment of inertia per unit length along field lines, and $V_{A, e x t}$ is the Alfvén speed in the external medium. You 
claimed that $\tau_{\|}=\tau_{I}$ is a "universal" relation. I would be a bit cautious about such a statement. In both our work and in your work just described, the field lines threading the rotator pass into the external medium and remain regular over distances "size of the rotator. If the flux tubes bend significantly near the rotator in the $(r, z)$ plane in the axisymmetric geometry we are using, then the torsional Alfvén waves, as they travel along curved field lines, will generate a velocity field which is not represented by a pure rotation, as assumed in your calculation. Thus, an important assumption will break down and consequently the conclusion will have to be modified. Altogether then, our result $\tau_{\|}=\tau_{I}$, which your calculation confirmed in a somewhat different geometry from that which we used, is useful and relatively general for an important class of aligned rotators, but one can hardly call that result universal on the basis of either our previous calculations or your present paper.

HEILES: One assumes flux freezing during the contraction phase. Instead, can the field be generated during the collapse by dynamo-type processes? If so, one might never have a cloud contracting to form a star in a field-free manner.

MESTEL: I think the problem is not to explain why a star has magnetic flux, but rather why so little of the galactic flux threading the original parent cloud survives trapped in the star. I agree that once most of this primeval flux has leaked out, dynamo action - yielding a very low $\mathrm{F} / \sqrt{\mathrm{GM}}$ - may subsequently occur, e.g. in an accretion disk in the later stages of star formation. 